\title{
Cinética de la Transesterificación del Aceite de Palma con Metóxido de Calcio
}

\author{
The Kinetic Palm Oil Transesterification with a Calcium Methoxide
}

\author{
J. C. Cardozo ${ }^{\mathrm{a}, *}$ \\ Rolando Barrera Zapata ${ }^{\mathrm{b}}$ \\ S. A. Giraldo ${ }^{\mathrm{c}}$
}

Recepción: 10-jul-13

Aceptación: 31 -oct-13

\section{Resumen}

Se proponen dos modelos cinéticos para la transesterificación del aceite de palma con metanol utilizando óxido de calcio; el primero incluye la adsorción del alcohol y del aceite sobre el catalizador mediante mecanismos Langmuir-Hinshelwood, y el segundo considera solo la adsorción del alcohol a través de mecanismos Eley-Rideal. Para deducir los modelos propuestos se estudió el efecto de la variación de la relación molar alcohol/aceite (4.9-8.9), del\%p/p de catalizador (0.3-0.7) y de la temperatura (328-338 K) sobre la velocidad de reacción. Los parámetros cinéticos se determinaron con el método de velocidades iniciales $(t<30 \mathrm{~min}$.), y para validar los modelos se utilizaron datos tomados para tiempos de reacción mayores (hasta $240 \mathrm{~min}$.). Se encontró que los modelos propuestos representan aceptablemente los datos experimentales con suma del cuadrado de residuales $<0.045$ y aportan elementos al entendimiento del fenómeno catalítico al introducir conceptos de adsorción/desorción en la cinética de la reacción.

Palabras clave: Aceite de palma, Cinética heterogénea, Mecanismo de reacción, Mecanismos LangmuirHinshelwood, Mecanismos Eley-Rideal, Transesterificación, Óxido de calcio.

\begin{abstract}
In this study, two new kinetic models for the palm oil transesterification with methanol, by using calcium oxide, are proposed. The first model, which includes the alcohol and the oil's adsorption on the catalyst, is carried out by the Langmuir-Hinshelwood mechanisms, while the second model only considers the alcohol adsorption on the solid, through the Eley-Rideal mechanisms. The Kinetic parameters were determined, by using the initial reaction rates method $(t<30 \mathrm{~min}$ ), while the data taken for longer times (until $240 \mathrm{~min}$ ) were used for the models validation. For the proposed models' deduction, it was studied the alcohol/oil molar ratio's variation effect (4.9-8.9), of the catalyst \%w/w (0.3-0.7) and the temperature (328-338 K) on the reaction speed. The kinetic parameters were determined using the initial reaction rates method $(t<30$ $\mathrm{min}$ ), while the data taken for longer times (until $240 \mathrm{~min}$ ) were used for models validation. It was found

\footnotetext{
${ }^{\mathrm{a}}$ Universidad Industrial de Santander, Bucaramanga, Santander.

${ }^{\mathrm{b}}$ Grupo CERES, Facultad de Ingeniería, Universidad de Antioquia, UdeA, Medellín, Colombia.

${ }^{c}$ Centro de Investigaciones en Catálisis, Escuela de Ingeniería Química, Facultad de Ingenierías Fisicoquímicas, Universidad Industrial de Santander, Bucaramanga, Santander.

*Correo electrónico: juancardozo_0121@ @hotmail.com
} 
that the proposed models represent, an acceptable experimental data, according to the squared residuals' sum $<0.045$. The models contribute to this catalytic system understanding, by introducing the adsorption/desorption concepts, on the reaction kinetics.

Key words: Calcium Oxide Eley-Rideal Mechanisms, Heterogeneous Kinetic, Langmuir-Hinshelwood Mechanisms, Palm Oil, Reaction Mechanism

\section{Introducción}

Desde antaño, la demanda energética se ha suplido a partir de combustibles de origen fósil, tales como petróleo, carbón y gas natural, pero en las últimas décadas se ha elevado progresivamente la preocupación por las emisiones que estos generan, dado que son nocivas para el medioambiente; esto ha llevado a la sociedad moderna a buscar e implementar nuevas tecnologías que garanticen su sostenibilidad y que al mismo tiempo sean técnicamente factibles, económicamente competitivas y ambientalmente amigables.

Para disminuir el consumo de combustibles de origen no renovable, el biodiésel aparece como alternativa interesante, dada su viabilidad tecnológica, financiera y ambiental, que lo perfilan como sustituto parcial o total del diésel [1,3]. En este contexto, la reacción de transesterificación ha sido ampliamente estudiada [4, 5]; con ella, a partir de aceite de origen vegetal se obtienen bio-ésteres, por el intercambio del grupo alcoxi de un éster por otro alcohol. Particularmente, los estudios relacionados con la cinética de la transesterificación de aceites se han caracterizado por tener dos tipos de enfoques complementarios entre sí: el empírico, donde a partir de datos experimentales se busca correlacionar el orden de reacción y demás parámetros para una expresión cinética dada, y el teórico, donde se plantea un mecanismo para la reacción, y a partir del mismo se deriva una expresión cinética [6, 7]. En cualquier caso, la validación experimental de la cinética propuesta es fundamental para establecer un modelo predictivo que pueda usarse en el diseño de reactores.

Los primeros estudios y fundamentos de estudios recientes $[8,9]$ muestran que en la transesterificación del aceite de palma con alcoholes se obtienen ésteres grasos y glicerina. Debido a que el aceite está compuesto por triglicéridos, se tienen reacciones intermedias, consecutivas e inmediatas a diglicéridos y monoglicéridos para obtener finalmente 3 moles de ésteres $\left(\mathrm{RCOOCH}_{3}\right.$ o FAMEs -sigla de Fatty Acid Methyl Esters-). Estequiométricamente, la reacción de transesterificación se representa por la ecuación (1).

$$
\text { Aceite }+3 R^{\prime} O H \leftrightarrow 3 F A M E+\text { Glicerina }
$$

A partir de la estequiometría de la reacción es posible determinar las distintas relaciones de velocidad de aparición y desaparición de reactivos y productos, ecuación (2) [7].

$$
-\frac{r_{A}}{1}=-\frac{r_{O H}}{3}=\frac{r_{F A M E}}{3}=\frac{r_{G}}{1}
$$

Vujicic y colaboradores [10] estudiaron la cinética de la transesterificación del aceite de girasol refinado utilizando temperaturas entre 60 y $120^{\circ} \mathrm{C}$, presiones entre 1 y 15 bar, relación molar metanol/aceite de 6:1 y $1 \%$ en peso de catalizador $\mathrm{CaO}$, en un sistema discontinuo agitado a $200 \mathrm{rpm}$. Concluyeron que la cinética de la reacción se describe adecuadamente por una expresión seudohomogénea de primer orden, observando además limitaciones difusionales externas e internas al incrementar la temperatura de reacción.

Diversos estudios acerca de la actividad del óxido de calcio como catalizador heterogéneo muestran que su uso es favorable en la transesterificación, señalando que la activación previa del $\mathrm{CaO}$ con metanol aumenta la conversión a metil ésteres del aceite de palma por medio de la formación del ión metóxido sobre la superficie del catalizador; además, el catalizador resulta estable para la reacción al no evidenciarse su desactivación hasta en 20 reutilizaciones $[11,15]$.

El recurrente uso de expresiones seudohomogéneas para describir la transesterificación de aceites vegetales $[10,16]$ se fundamenta en la relativa simplicidad de este tipo de expresiones y en la dificultad que supone el estudio y validación de un mecanismo para la reacción en el sistema heterogéneo $[17,18]$. Aunque los datos experimentales pueden ajustarse adecuadamente a un modelo seudohomogéneo, para la ingeniería del proceso puede ser deseable utilizar expresiones que también describan el efecto de la 
adsorción y desorción de las especies a lo largo de la reacción, ampliando el espectro fenomenológico cubierto por el modelo e incrementando de esta forma su capacidad predictiva.

En el mecanismo propuesto para este tipo de reacciones [16, 17], el ión metóxido que se une a la superficie del catalizador ataca al grupo carbonilo de la molécula de triglicéridos (etapa 1), dando formación a un intermedio tetraédrico (etapa 2). Posteriormente, el intermedio se reorganiza para formar un anión diglicérido y un mol de éster metílico (etapa 3). El anión diglicérido se estabiliza por un protón de la superficie del catalizador para formar diglicérido y regenerar el catalizador. El ciclo continúa hasta que los tres centros carbonilo en el triglicérido han sido atacados por iones metóxido, produciendo un mol de glicerol y tres moles de ésteres metílicos. Si bien los reportes de la literatura coinciden en que al comienzo de la reacción catalizada con $\mathrm{CaO}$ se forma una fase activa, consistente en un ión metóxido que da paso a la formación de intermedios tetraédricos, persisten dudas sobre el mecanismo de reacción que implica la formación y ruptura de dichos intermedios $[16,18]$.

En este trabajo se parte de los mecanismos heterogéneos propuestos para la transesterificación de aceite de palma con metanol utilizando $\mathrm{CaO}$, y se introducen diversas suposiciones apoyadas en las observaciones experimentales que permiten formular nuevas expresiones cinéticas. Haciendo uso de un adecuado diseño experimental, se correlacionan los parámetros cinéticos de dichas expresiones (constante específica de reacción, factor preexponencial y energía de activación), así como las constantes de equilibrio de adsorción correspondientes. La capacidad predictiva de los modelos propuestos se valida experimentalmente. Se espera que los resultados de este trabajo contribuyan a un mejor entendimiento de este sistema catalítico, ya que incluyen la descripción de fenómenos de adsorción y desorción sobre la superficie del catalizador.

\section{Experimental}

\subsection{Diseño experimental}

Las condiciones para la transesterificación de aceite de palma con metanol utilizando óxido de calcio en un reactor discontinuo (tabla 1) fueron tomadas de la literatura [14]. Estas condiciones describen el sistema experimental que en adelante se denomina "sistema de reacción estándar". De acuerdo con la literatura [14], bajo dichas condiciones se garantiza la ausencia de limitaciones difusionales, y es posible realizar mediciones de la cinética intrínseca de la reacción.

Para este estudio se definieron los factores relación molar alcohol/aceite (cantidad de alcohol y aceite), cantidad de catalizador y temperatura de reacción, considerando que son fácilmente medibles o fáciles de correlacionar en el sistema de reacción. Se espera que la velocidad de reacción presente variabilidad ante fluctuaciones de estos factores. $\mathrm{La}$ correlación entre las moles y la masa del alcohol y el aceite usado en los experimentos se estimó con base en su peso molecular, utilizando para el aceite el peso molecular promedio de acuerdo con su composición, que se detalla posteriormente en la sección "Montaje experimental". Dado que el objetivo de la experimentación fue conocer la influencia de cada una de las variables en la velocidad de reacción, se utilizó un diseño experimental de un factor a la vez [19]. Para el diseño experimental se utilizaron dos niveles superiores y dos niveles inferiores a los valores estándar para las variables cantidad de alcohol, aceite y catalizador, mientras que para la variable temperatura se utilizó un nivel superior y uno inferior. Para todos los experimentos se utilizó un volumen total de reacción $\approx 50 \mathrm{ml}$. La descripción de niveles para cada factor y su correspondiente valor se presentan en la tabla 2.

Tabla 1. Condiciones de activación y reacción [14].

\begin{tabular}{lc}
\hline Parámetro & Valor \\
\hline Tiempo de activación & $1.5 \mathrm{~h}$ \\
Temperatura de activación & Ambiente \\
Relación molar metanol/aceite & 6.9 \\
\% Catalizador (p) & 0.5 \\
Temperatura de reacción & $333 \mathrm{~K}$ \\
Tiempo de reacción & $4 \mathrm{~h}$ \\
Agitación & $770 \mathrm{rpm}$ \\
\hline
\end{tabular}

Tabla 2. Niveles y factores del diseño experimental.

\begin{tabular}{lccccc}
\hline NIVEL FACTOR & -2 & -1 & 0 & 1 & 2 \\
\hline Metanol $(\mathrm{g})$ & 15.31 & 17.01 & 18.62 & 19.96 & 21.13 \\
Aceite $(\mathrm{g})$ & 26.32 & 24.39 & 22.73 & 21.28 & 20.00 \\
Catalizador $(\mathrm{g})$ & 0.125 & 0.166 & 0.207 & 0.247 & 0.288 \\
Temperatura & & 328.0 & 333.0 & 338.0 & \\
de reacción $(\mathrm{K})$ & & & & & \\
\hline
\end{tabular}


En la tabla 3 se muestra la matriz de experimentos con los valores correspondientes de los niveles de variables de la tabla 2 (temperatura y cantidad de metanol, aceite y catalizador). Con esta matriz de experimentos se evaluó el efecto de la concentración de cada una de las especies (aceite, alcohol y catalizador) en la velocidad de reacción. Se destaca que los experimentos $3,8,13$ y 17 (tabla 3 ) utilizan la misma cantidad de aceite, metanol y catalizador, y la misma temperatura, esto porque corresponden a la reacción estándar y 3 réplicas, cuyos resultados se utilizaron para establecer la incertidumbre en la determinación de la conversión de aceite en el sistema de reacción. Los experimentos 16 y 18 (tabla 3) se realizaron con las mismas cantidades de metanol, aceite y catalizador que se usaron en la reacción estándar, pero a diferentes temperaturas. Los resultados de estos experimentos se utilizaron para determinar los parámetros cinéticos, energía de activación y factor preexponencial [7].

Tabla 3. Matriz del diseño experimental.

\begin{tabular}{ccccc}
\hline Experimento & Aceite & Metanol & $\mathrm{CaO}$ & $\mathrm{T}$ \\
\hline 1 & -2 & 0 & 0 & 0 \\
2 & -1 & 0 & 0 & 0 \\
$3 *$ & 0 & 0 & 0 & 0 \\
4 & 1 & 0 & 0 & 0 \\
5 & 2 & 0 & 0 & 0 \\
6 & 0 & -2 & 0 & 0 \\
7 & 0 & -1 & 0 & 0 \\
$8 *$ & 0 & 0 & 0 & 0 \\
9 & 0 & 1 & 0 & 0 \\
10 & 0 & 2 & 0 & 0 \\
11 & 0 & 0 & -2 & 0 \\
12 & 0 & 0 & -1 & 0 \\
$13 *$ & 0 & 0 & 0 & 0 \\
14 & 0 & 0 & 1 & 0 \\
15 & 0 & 0 & 2 & 0 \\
16 & 0 & 0 & 0 & -1 \\
$17 *$ & 0 & 0 & 0 & 0 \\
18 & 0 & 0 & 0 & 1 \\
\hline *Experimentos estándar. & & &
\end{tabular}

\subsection{Montaje experimental}

Tanto para la activación del catalizador como para la reacción de transesterificación se utilizó un sistema de reacción discontinuo, compuesto por un balón de tres bocas con capacidad para $100 \mathrm{ml}$, con sistema de condensación (reflujo), muestreo, control de temperatura y agitación magnética, similar al utilizado por Gómez [14].
Formación del metóxido de calcio (Activación). La activación del catalizador se realizó según el procedimiento reportado [14]. El metanol (pureza 99.9\%, Merck) se puso en contacto con el $\mathrm{CaO}$ pulverizado (diámetro de partícula promedio $280 \mu \mathrm{m}$ ) sin calcinar, en las proporciones definidas para cada experimento (tablas 1 y 3). La mezcla se mantuvo bajo agitación magnética (770 rpm) durante $1.5 \mathrm{~h}$ a temperatura ambiente (promedio $301 \mathrm{~K}$ ).

Reacción de transesterificación. La composición de ácidos grasos en el aceite de palma, suministrado por la empresa Saceites S.A.S., fue: $43.2 \%$ de ácido palmítico, $42 \%$ de ácido oleico, $9.2 \%$ de ácido linoleíco, $4 \%$ de ácido esteárico y $1.6 \%$ de trazas de otros ácidos grasos. Dado que a temperatura ambiente el aceite solidifica, se precalentó (aproximadamente 5 minutos a $310 \mathrm{~K}$ ) para tenerlo en fase fluida. La cantidad correspondiente a cada experimento se adicionó al sistema una vez finalizada la etapa de activación y se llevó a la temperatura de reacción, la cual se controló con $\pm 1{ }^{\circ} \mathrm{C}$ de precisión, utilizando una plancha de agitación con control de temperatura (WISESTIR MSH200). Del sistema de reacción se extrajeron muestras $(\approx 0.5 \mathrm{ml})$ a los 5 , $10,15,20,25,30,60,120,180$ y 240 min., que posteriormente se analizaron por cromatografía de gases (CG).

\subsection{Preparación y análisis de muestras de reacción}

Las muestras de reacción se decantaron y se analizó la capa menos densa (rica en metil ésteres) mediante CG. Para el análisis cromatográfico se disolvieron $20 \mu \mathrm{l}$ de la capa menos densa (metil ésteres) en $800 \mu \mathrm{l}$ de n-hexano (pureza $>96 \%$, Merck) y $2 \%$ en peso de estándar interno (dodecano pureza $>99 \%$, Sigma-Aldrich). Se usó un cromatógrafo HP 6890 equipado con una columna capilar HP-INNOWAX, $30 \mathrm{~m} \times 0.32 \mathrm{~mm} \times 0.25 \mu \mathrm{m}$, con detector FID e inyector automático. La rampa de temperatura del horno fue: $90{ }^{\circ} \mathrm{C}$ (4 min.), seguida de una rampa de $50{ }^{\circ} \mathrm{C} / \mathrm{min}$. hasta $220{ }^{\circ} \mathrm{C}$ (4 min.) y finalmente una rampa de $10{ }^{\circ} \mathrm{C} / \mathrm{min}$. hasta $230^{\circ} \mathrm{C}(9 \mathrm{~min}$.). Para cuantificar los metil ésteres (FAMEs) se utilizó una curva de calibración realizada con una mezcla de metil ésteres conocidos (Supelco).

Determinación de la conversión de aceite. Una vez determinada por CG la concentración de FAMEs 
en la muestra, y considerando los factores de dilución correspondientes, se calculó la concentración de FAMEs. A partir de esta información se estimó la cantidad de aceite que reaccionó, usando la estequiometría de la reacción (ecuación 1).

Incertidumbre en la determinación de la conversión de aceite. Dada la complejidad en la cuantificación de cada una de las posibles fuentes de error asociadas a la investigación, se optó por determinar una incertidumbre combinada [20,21]. Para ello se realizaron el experimento estándar y 3 réplicas (experimentos $3,8,13$ y 17 , tabla 3 ) y se analizaron el promedio y la desviación estándar para la conversión de aceite en cada tiempo de reacción analizado.

Evaluación del efecto del cambio de volumen en la mezcla de reacción. Para evaluar el efecto del cambio de volumen de la mezcla de reacción con la extracción sucesiva de muestras en el tiempo se realizaron un conjunto de experimentos adicionales (experimentos a volumen constante), donde se preparó la mezcla de reacción estándar y se tomó una única muestra después de un tiempo de reacción fijo. Posteriormente, los resultados se compararon con la conversión de aceite determinada para el mismo tiempo de reacción en experimentos donde se efectuó la toma sucesiva de muestras para diferentes tiempos.

\subsection{Modelos cinéticos propuestos}

Modelo a partir de mecanismos LangmuirHinshelwood (LH). Los mecanismos de reacción propuestos en este estudio se apoyan en la literatura descrita en la introducción [17], los cuales se simplificaron suponiendo que el ataque nucleofílico se da simultáneamente en cada uno de los 3 grupos carbonilos de la molécula de triglicérido, generando un intermedio tetraédrico del cual se obtienen las 3 moléculas de FAMEs y el anión glicerol a través del mecanismo descrito en las ecuaciones (3-6); es decir, para este mecanismo no se considera la formación de diglicéridos ni monoglicéridos, sino que la transformación de triglicéridos a metil ésteres se da en un solo paso. Por tal razón, en el mecanismo propuesto la etapa de reacción (ecuación 5) se considera irreversible.

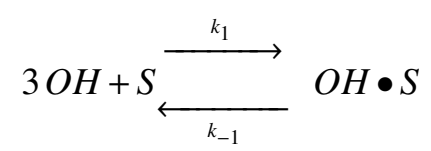

$$
\begin{aligned}
& \mathrm{OH}+\mathrm{S}+A_{k_{-2}}^{\stackrel{k_{2}}{\longrightarrow}} A \bullet \mathrm{OH} \bullet \mathrm{S} \\
& A \bullet \mathrm{OH} \bullet S \stackrel{k_{3}}{\longrightarrow} G \bullet S+3 F \\
& G \bullet S \underset{k_{-4}}{\stackrel{k_{4}}{\longleftrightarrow}} G+S
\end{aligned}
$$

Donde $\mathrm{OH}$ representa la especie alcohol; $\mathrm{S}$, los sitios catalíticos disponibles; $\mathrm{OH} \bullet \mathrm{S}$, las especies intermedias formadas por la adsorción del alcohol sobre el catalizador (especie catalíticamente activa); A, el aceite; $A \bullet O H \bullet S$, las especies intermedias formadas por la adsorción del aceite sobre la especie catalíticamente activa; G, la glicerina, y F, los FAMEs.

Utilizando la hipótesis del estado pseudoestacionario, donde se asume que la velocidad neta (aparición y desaparición) de las especies intermedias es cero [7], se plantean las ecuaciones (7-9), con las cuales se hace posible expresar la concentración de las especies intermedias en términos de las concentraciones conocidas (aceite, alcohol y catalizador).

$$
\begin{aligned}
-r_{O H} \bullet S & =0=k_{1}[O H]^{3}[S]-k_{-1}[O H \bullet S] \\
& -k_{2}[O H \bullet S][A]+k_{-2}[A \bullet O H \bullet S]
\end{aligned}
$$

$$
\begin{aligned}
-r_{A} \bullet \mathrm{OH} S & =0=k_{2}[O H \bullet S][A] \\
-k_{-2}[A \bullet O H \bullet S]-k_{3}[A \bullet O H \bullet S] & \\
-r_{G} \bullet S & =0=k_{3}[A \bullet O H \bullet S] \\
- & k_{4}[G \bullet S]+k_{-4}[G][S]
\end{aligned}
$$

Con: $[\mathrm{OH}],[\mathrm{A}],[\mathrm{G}],[\mathrm{S}]$ = Concentración de alcohol, aceite, glicerina y sitios disponibles del catalizador, respectivamente.

Suponiendo que la etapa de reacción (ecuación 5) es la etapa controlante, como sucede con el $75 \%$ de las reacciones catalíticas [7], la velocidad de reacción del aceite está dada por la ecuación (10); implicando además que la magnitud de la constante $k_{3}$ es mucho menor que la magnitud de las demás constantes.

$$
-r_{A}=k_{3}[A \bullet O H \bullet S]
$$


De las ecuaciones (7-9) se tiene:

$$
\begin{aligned}
{[\mathrm{OH} \bullet S] } & =K_{O H}[\mathrm{OH}]^{3}[S] \\
{[A \bullet O H \bullet S] } & =K_{O H} K_{A}[A][O H]^{3}[S] \\
{[G \bullet S] } & =\frac{k_{3} K_{O H} K_{A}}{k_{4}}[A][H]^{3}[S] \\
& +\frac{1}{K_{G}}[G][S]
\end{aligned}
$$

Donde las constantes, $K_{O H}=\frac{k_{1}}{k_{-1}}, K_{A}=\frac{k_{2}}{k_{-2}}, K_{G}=$ $\frac{k_{4}}{k_{-4}}$, representan las constantes de equilibrio de adsorción de alcohol, aceite y glicerina, respectivamente.

La concentración de sitios libres $[S]$ se puede obtener de la concentración total de sitios en el catalizador, $C_{T}$, la cual estará conformada por los sitios libres y los sitios donde se adsorben las especies, ecuación (14).

$$
C_{T}=[S]+[O H \bullet S]+[A \bullet O H \bullet S]+[G \bullet S]
$$

Sustituyendo las ecuaciones (11)-(13) en (14), y despejando la concentración de sitios disponibles, $[S]$, se tiene:

$$
[S]=\frac{C_{T}}{\left(1+K_{O} H[O H]^{3}+K_{O} H K_{A}[A][O H]^{3}+\left(k_{3} K_{O} H K_{A}\right) / k_{4}[A][O H]^{3}+k_{-4} / k_{4}[G]\right.}
$$

Sustituyendo las ecuaciones (11-13) y (15) en (10), se obtiene la Ecuación cinética derivada del mecanis- mo Langmuir-Hinshelwood, ecuación (16) (Modelo LH).

$$
-r_{A}=\frac{k_{3} K_{O} H K_{A}[A][O H]^{3} C_{T}}{1+K_{O} H[O H]^{3}+K_{O} H K_{A}[A][O H]^{3}+\left(k_{3} K_{O} H K_{A}\right) / k_{4}[A][O H] 3+(1) / K_{G}[G]}
$$

Modelo a partir de mecanismos Eley-Rideal (ER). Para el segundo mecanismo propuesto, en las ecuaciones (17-19) se supone que no ocurre la adsorción del aceite sobre la especie intermedia formada por la adsorción del alcohol en el catalizador $(\mathrm{OH} \bullet \mathrm{S})$, sino que una vez el aceite (A) entra en contacto con la especie activa (metóxido), se obtienen los productos, FAMEs (F) y glicerina $(\mathrm{G})$, la cual posteriormente se desorbe para regenerar el sitio catalítico. Suponiendo nuevamente que la reacción (ecuación 18) es la etapa controlante, se obtiene la

ecuación (20). (Modelo ER).

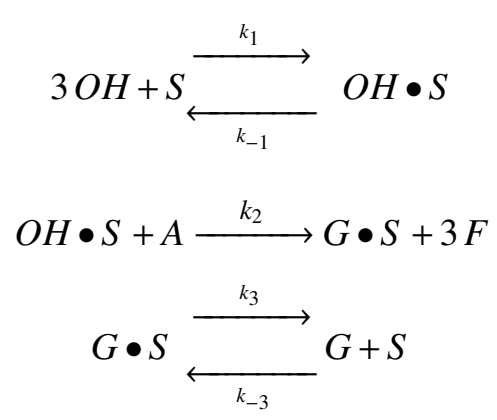

Donde las constantes de equilibrio de adsorción del alcohol y la glicerina se definen por $K_{O H}=\frac{k_{1}}{k_{-1}}$ y $K_{G}=\frac{k_{3}}{k_{-3}}$, respectivamente.

$$
-r_{A}=\frac{k_{2} K_{O} H[A][O H]^{3} C_{T}}{1+K_{O} H[O H]^{3}+\left(k_{2} K_{O} H\right) / k_{3}[A][O H]^{3}+(1) / K_{G}[G]}
$$

Correlación de parámetros cinéticos. Los parámetros correspondientes a las constantes de reacción y a las constantes de equilibrio de adsorción de los modelos propuestos se estimaron por regresión no 
lineal de los datos experimentales de velocidad de reacción inicial con la herramienta Solver de Microsoft Excel. Se aplicó un método evolutivo que utiliza algoritmos genéticos en la búsqueda del óptimo para el problema definido utilizando una precisión de $1 \times 10^{-9}$, máximo de 1000 iteraciones, $300 \mathrm{~s} \mathrm{y}$ especificando límites para los parámetros de acuerdo con los reportes de la literatura [10, 16, 22]. El parámetro CT se fijó de acuerdo con los datos experimentales; así, por ejemplo, para la reacción estándar se estimó en un valor de $7.37 \times 10^{-2} \mathrm{~mol} / \mathrm{l}$. Por otro lado, con los resultados de las reacciones 8,16 y 18 , de la Tabla 3 (variación de la temperatura de reacción), se estimaron los parámetros cinéticos energía de activación, $E_{a}$, y factor preexponencial de Arrhenius, A; para esto se utilizó la metodología propuesta en la literatura [7], linealizando la Ecuación de Arrhenius, graficando $\ln k$ Vs. $1 / T$ y determinando la pendiente de la recta obtenida como $-E_{a} / R$, con $R=$ constante universal de los gases.

Validación y comparación de los modelos. Para validar los modelos y comparar su capacidad pre- dictiva en la transesterificación de aceite de palma con metóxido de Ca, se utilizó la totalidad de los datos experimentales (hasta $240 \mathrm{~min}$. de reacción) y no solo los datos de velocidad de reacción inicial (primeros 20 min. de reacción) que se usaron para correlacionar los parámetros. Para ello, las expresiones cinéticas (ecuaciones 16 y 20) se llevaron a la ecuación de diseño de un reactor por lotes, suponiendo volumen constante y considerando la estequiometría de la reacción [7]. Adicionalmente, se expresó la concentración de las especies presentes en el sistema de reacción en términos de la concentración inicial del reactivo límite (aceite) y la conversión $(\mathrm{X})$, ecuaciones (21-24), que al sustituirlas en la ecuación (16) (modelo LH) generan la ecuación (25).

$$
\begin{gathered}
C_{A}=C_{A O}(1-X) \\
C_{O H}=C_{A O}(\theta-3 X) \\
C_{F}=C_{A O}(3 X) \\
C_{G}=C_{A O}(X)
\end{gathered}
$$

$$
-r_{A}=\frac{k_{3} K_{O H} K_{A} C_{A O}^{3}(1-X)(\theta-3 X)^{3} C_{T}}{1+K_{A} K_{O H} C_{A O}^{4}(1-X)(\theta-3 X)^{3}\left(1+\frac{k_{3}}{k_{4}}\right)+C_{A O}^{3} K_{O H}(\theta-3 X)^{3}+\frac{1}{K_{G}} C_{A O}(X)}
$$

\subsection{Incertidumbre en la determinación de la conversión de aceite}

Con los resultados de la determinación de la conversión de aceite en las réplicas del experimento estándar (experimentos 3, 8, 13 y 17, tabla 3), se estimó el promedio y la desviación estándar para la conversión de aceite en cada tiempo de reacción analizado (tabla 4).

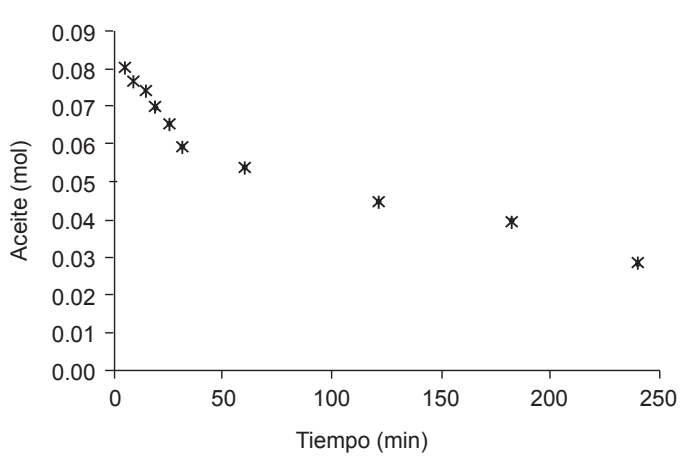

Figura 1. Evolución de la reacción estándar. 
Tabla 4. Desviación estándar de la conversión de aceite.

\begin{tabular}{ccc}
\hline $\begin{array}{c}\text { Tiempo } \\
(\mathrm{min})\end{array}$ & $\begin{array}{c}\text { Conversión de } \\
\text { Aceite Promedio }\end{array}$ & $\begin{array}{c}\text { Desviación } \\
\text { Estándar, \% }\end{array}$ \\
\hline 0 & 0.000 & 0.0 \\
5 & 0.024 & 2.3 \\
10 & 0.059 & 3.2 \\
15 & 0.091 & 3.4 \\
20 & 0.138 & 3.4 \\
25 & 0.177 & 4.4 \\
30 & 0.242 & 4.7 \\
60 & 0.308 & 5.0 \\
120 & 0.406 & 5.6 \\
180 & 0.513 & 5.8 \\
240 & 0.597 & 5.8 \\
\hline
\end{tabular}

En la tabla 4 se observa que la máxima desviación (alrededor del 6\%) se encuentra para los mayores tiempos de reacción, lo cual puede explicarse por los cambios (particularmente en volumen) que presenta el sistema a partir de la extracción sucesiva de muestras en el tiempo. Aun así, se observa que para las velocidades de reacción iniciales, utilizadas posteriormente para la correlación de datos de este estudio, la desviación es alrededor de $3 \%$. Se asume que en este error se incluyen simultáneamente todas las posibles fuentes de incertidumbre presentes en la experimentación y análisis [20,21].

\subsection{Validación de la suposición de volumen constante}

En el peor escenario, para la última muestra tomada el volumen de reacción disminuyó en un 10\% del inicial. Considerando que en la determinación de parámetros de las expresiones cinéticas se utilizan valores de velocidad de reacción inicial, para estos tiempos de reacción el volumen inicial no ha alcanzado variaciones superiores al $2 \%$, y por lo tanto es válido suponer un volumen constante para el análisis de resultados. Adicionalmente, esta suposición se validó comparando los resultados de experimentos independientes (condiciones estándar) para cada tiempo de reacción (es decir, a volumen constante) con la conversión de aceite determinada para el mismo tiempo de reacción en experimentos donde se efectuó la toma sucesiva de muestras para diferentes tiempos. En la figura 2 se observa la similitud para todos los datos dentro de la incertidumbre definida.

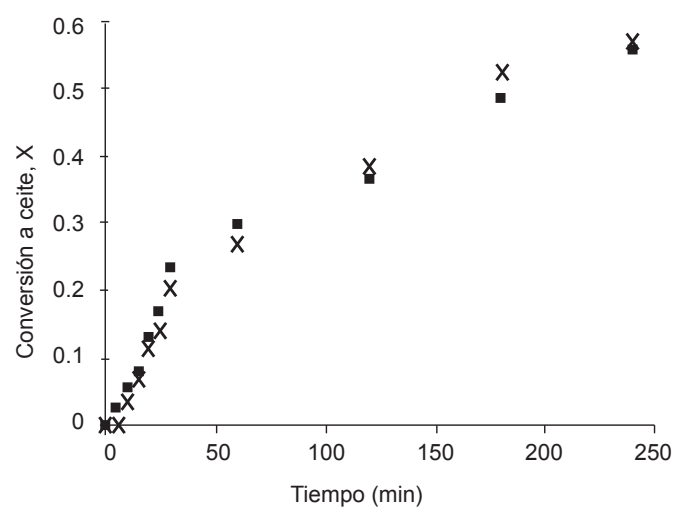

Figura 2. Comparación de la conversión de aceite en el experimento estándar (匹) con experimentos independientes a volumen constante $(\mathrm{x})$.

\subsection{Validación y comparación de los modelos}

Las velocidades de reacción iniciales se determinaron a partir de la cantidad de moles de aceite en el tiempo para cada reacción (figura 1). Dado el comportamiento lineal para los primeros minutos de la reacción, la curva se ajustó a una línea recta cuya pendiente $(\mathrm{dn} \mathrm{A} / \mathrm{dt})$ puede asumirse como la velocidad de reacción inicial. Los valores de la velocidad inicial de todas las reacciones se presentan en la tabla 55.

La correlación de parámetros para los modelos LH (ecuación 16) y ER (ecuación 20) se realizó con la herramienta Solver de Microsoft Excel. Los resultados de las correlaciones se muestran en la tabla 6.

Tabla 5. Velocidad de reacción inicial para cada experimento.

\begin{tabular}{cccc}
\hline Reacción & $\begin{array}{c}\text { Velocidad } \\
\text { Inicial } \\
{[\mathrm{mol} /(\mathrm{L} \mathrm{min} .)]}\end{array}$ & Reacción & $\begin{array}{c}\text { Velocidad } \\
\text { Inicial } \\
{[\mathrm{mol} /(\mathrm{L} \mathrm{min} .)]}\end{array}$ \\
\hline 1 & -0.0617 & 10 & -0.1089 \\
2 & -0.5015 & 11 & -0.2049 \\
3 & -0.1031 & 12 & -0.1504 \\
4 & -0.1034 & 13 & -0.1221 \\
5 & -0.0453 & 14 & -0.0650 \\
6 & -0.1476 & 15 & -0.1272 \\
7 & -0.1207 & 16 & -0.1371 \\
8 & -0.1047 & 17 & -0.1897 \\
9 & -0.4069 & 18 & -0.1429 \\
\hline
\end{tabular}

\subsection{Correlación de parámetros cinéticos}

Las ecuaciones (25) y (26) se solucionaron numéricamente con el método de Runge-Kutta de orden 4 [23], utilizando los parámetros de la Tabla 6. Los resultados se muestran en la figura 3 . 
Tabla 6. Parámetros correacionados para los modelos propuestos.

\begin{tabular}{|c|c|c|}
\hline \multicolumn{3}{|c|}{ Modelo LH (ecuación 16) } \\
\hline Parámetro & Valor & Unidades \\
\hline$k_{3}$ & 0.066 & $\operatorname{Min}^{-1}$ \\
\hline$K_{O H}$ & 0.99 & $\mathrm{~L}^{3} / \mathrm{mol}-1^{3}$ \\
\hline$K_{A}$ & $836,387.3$ & $\mathrm{~L} / \mathrm{mol}$ \\
\hline$k_{3} / k_{4}$ & $1.41 \times 10^{-8}$ & Adim* \\
\hline$K_{G}$ & 605.1 & $\mathrm{~mol} / \mathrm{L}$ \\
\hline \multicolumn{3}{|c|}{ Modelo ER (ecuación 20) } \\
\hline Parámetro & Valor & Unidades \\
\hline$k_{2}$ & 0.056 & $\left(\right.$ L. $\left.\min ^{-1}\right) / \mathrm{mol}$ \\
\hline$K_{O H}$ & 390,4 & $\mathrm{~L}^{3} / \mathrm{mol}^{3}$ \\
\hline$k_{2} / k_{3}$ & $1 \times 10^{-6}$ & $\mathrm{~L} / \mathrm{mol}$ \\
\hline$K_{G}$ & $2,957.2$ & $\mathrm{~mol} / \mathrm{L}$ \\
\hline
\end{tabular}

Los parámetros cinéticos, energía de activación y factor preexponencial, correlacionados para los dos modelos, se muestran en la tabla 7, donde se observa una adecuada concordancia (en magnitud) con valores reportados para estos parámetros en la literatura para sistemas de reacción similares (energías de activación entre 21 y $51 \mathrm{KJ} / \mathrm{mol}[10,22]$ ).

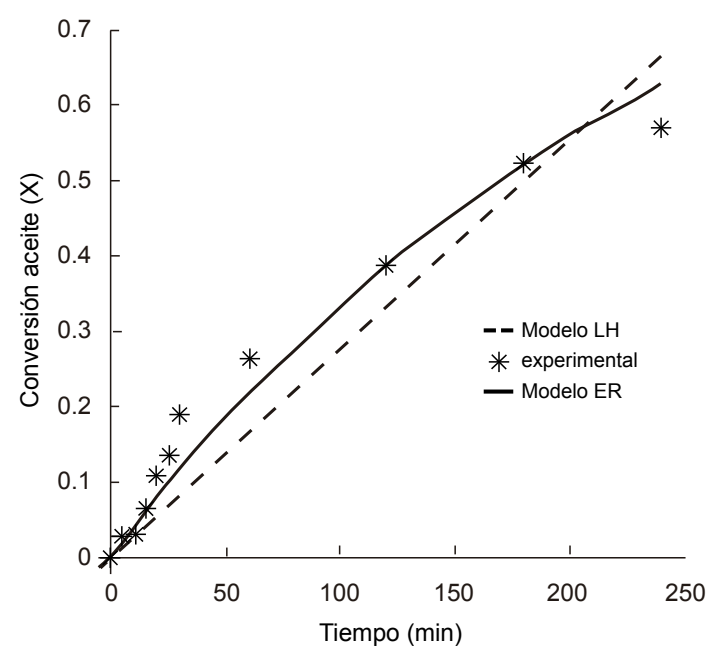

Figura 3. Comparación de la conversión estimada con el modelo LH, ecuación (25), (- -), el modelo ER, ecuación (26), (-) y los datos experimentales $(*)$.

Tabla 7. Parámetros cinéticos para cada uno de los modelos propuestos.

\begin{tabular}{ccc}
\hline Parámetro & Modelo & Modelo \\
& LH (ecuación 25) & ER (ecuación 26) \\
\hline$k$ & 0.066 & 0.056 \\
$E_{a}(\mathrm{Kj} / \mathrm{mol})$ & 53.162 & 53.844 \\
$\mathrm{~A}\left(\mathrm{~min}^{-1}\right.$ & $1.15 \times 10^{8}$ & $1,15 \times 10^{8}$ \\
\hline
\end{tabular}

Para comparar estadísticamente la precisión de los modelos en la representación de los datos expe- rimentales se determinó la suma de la diferencia de cuadrados entre los datos estimados por los modelos y los valores experimentales correspondientes. Los resultados se presentan en la tabla 8 .

Tabla 8. Diferencia de la suma de cuadrados para los modelos propuestos.

\begin{tabular}{cc}
\hline Modelo & $\begin{array}{c}\text { Diferencia de } \\
\text { Cuadrados (SCR) }\end{array}$ \\
\hline Modelo LH (ecuación 25) & 0.046 \\
Modelo ER (ecuación 26) & 0.015 \\
\hline
\end{tabular}

Para los dos modelos cinéticos que se propusieron en esta investigación se logró obtener una aceptable representación del sistema. De la figura 3 y la tabla 7 se observa una mejor representación de los datos experimentales con el modelo ER, en el cual se asume que el aceite no se adsorbe sobre los sitios catalíticos. De acuerdo con ese mecanismo (ecuaciones 17-19), podría inferirse que no es necesario un sistema de reacción que garantice el contacto prolongado entre el aceite y el catalizador activado (metóxido de calcio), y posiblemente reactores en continuo de lecho fijo o lecho fluidizado resulten efectivos para la eventual implementación del sistema a escala piloto o industrial. Se observa que existe una mejor descripción de las conversiones a tiempos cortos (figura 3), lo cual es justificable, ya que se usaron los datos de velocidad de reacción inicial para obtener los parámetros característicos de cada modelo. Para tiempos superiores a $60 \mathrm{~min}$. la correlación de los modelos evidencia diferencias con relación a los datos experimentales, en especial el modelo LH. Es importante considerar que la incertidumbre en los datos experimentales es mayor para los tiempos superiores.

\section{Conclusiones}

Se propusieron dos modelos cinéticos heterogéneos que representan en forma aceptable (SCR < $0,045)$ la transesterificación de aceite de palma con metanol, utilizando metóxido de Ca como catalizador, y a su vez ayudan a entender con mayor detalle el proceso catalítico, por incluir fenómenos de adsorción y desorción. Los mejores resultados se obtuvieron con modelos derivados del mecanismo EleyRideal. La validación de los parámetros cinéticos encontrados, a partir de las velocidades de reacción inicial, arrojó valores de constantes y parámetros cinéticos dentro de los intervalos reportados por la 
literatura; por lo tanto, los modelos propuestos pueden utilizarse para el modelado y simulación de la reacción o para el diseño de reactores que operen dentro de las condiciones definidas en este estudio.

\section{Agradecimientos}

Los autores agradecen a la Vicerrectoría de Investigación y Extensión de la UIS por el soporte financiero a través del proyecto "Aprovechamiento Energético de la Biomasa Residual”, código 5451.

\section{Referencias}

[1] A. Murugesan, C. Umarani, R. Subramanian, N. Nedunchezhian, "Biodiesel as an alternative fuel for diesel engines -A review-", Renew. Sust. Energ. Rev, vol. 13, pp. 653-662, 2009.

[2] S. Sumathi, S.P Chai., A.R. Mohamed, "Utilization of oil palm as a source of renewable energy in Malaysia", Renew. Sust. Energ. Rev, vol. 12, pp. 2404-2421, 2008.

[3] T. Silalertruksa, S. Bonnet, S.H. Gheewala, "Life cycle costing and externalities of palm oil biodiesel in Thailand", J. Clean. Prod, vol. 28, pp. 225-232, 2012.

[4] K.T. Tan, K.T. Lee, A.R. Mohamed, S. Bhatia, "Palm oil. Addressing issues and towards sustainable development", Renew. Sust. Energ. Rev, vol. 13, pp. 420-427, 2009.

[5] H. C. Ong, T. M. I. Mahlia, H. H. Masjuki, R. S. Norhasyima, "Comparison of palm oil, Jatrophacurcas and Calophylluminophyllum for biodiesel: A review", Renew. Sust. Energ. Rev, vol. 15, pp. 3501-3515, 2011.

[6] V. B. Veljkovic, O. S. Stamenkovic, Z. B. Todorovic, M. L. Lazic, D. U. Skala "Kinetics of sunflower oil methanolysis catalyzed by calcium oxide", Fuel, vol. 88, pp. 1554-1562, 2009.

[7] H. S. Fogler, Elementos de ingeniería de las reacciones químicas, 4 ed. EEUU: Prentice Hall, 2008.

[8] M. Mittelbach, B. Trathnigg, "Kinetics of Alkaline Catalyzed Methanolysis of Sunflower Oil”, Eur. J. Lipid. Sci. Technol, vol. 92, pp. 145?148, 2006.
[9] G. Vicente, M. Martínez, J. Aracil, A. Esteban, "Kinetics of sunflower oil methanolysis", Ind. Eng. Chem. Res, vol. 44, pp. 5447-5454, 2005.

[10] D. Vujicic, D. Comic, A. Zarubica, R. Micic, G. Boskovic, "Kinetics of biodiesel synthesis from sunflower oil over $\mathrm{CaO}$ heterogeneous catalyst”, Fuel, vol. 89, pp. 2054-2061, 2010.

[11] M. Becerra, A. Centeno, S. Giraldo, "Búsqueda de catalizadores sólidos básicos en reacciones de transesterificación para la producción de biodiesel”, Información Tecnológica, vol. 21, no. 4, pp. 57-66, 2010.

[12] M. Becerra, A. Centeno, S. Giraldo, "Triglyceride transesterification in heterogeneous reaction system with calcium oxide as catalyst", Rev. Fac. Ing. Univ. Antioquia, vol. 57, pp.7-13, 2011.

[13] M. Becerra, J. López, A. Centeno, S. Giraldo "Producción de biodiésel y glicerina limpia empleando catálisis básica heterogénea", Rev. Ion, Bucaramanga, vol. 21, no. 1, pp. 31-38, 2008.

[14] L. Gómez, Evaluación del óxido de calcio en la transesterificación del aceite de palma, "Trabajo de grado, Ingeniería Química", Bucaramanga, Colombia, Universidad Industrial de Santander, 2011. Disponible en http://tangara.uis.edu.co/biblioweb/tesis/2011/ 137915.pdf.

[15] X. Liu, X. Piao, Y. Wang, S. Zhu, H. He, "Calcium methoxide as a solid base catalyst for the transesterification of soybean oil to biodiesel with methanol", Fuel, vol. 87, pp. 1076-1082, 2008.

[16] N. C. OmTapanes, D. A. Gomes, J. W. de Mesquita, O. A. Antunes "Transesterification of Jatrophacurcas oil glycerides: Theoretical and experimental studies of biodiesel reaction", Fuel, vol. 87, pp. 2286-2295, 2008.

[17] M. L. Granados, M. D. Poves, D. M. Alonso, R. Mariscal, F. C. Galisteo, R. Moreno-Tost, J. Santamaría, J. L. Fierro, "Biodiesel from sunflower oil by using activated calcium oxide", Appl. Catal. B. Environ, vol. 73, pp. 317-326, 2007.

[18] P. L. Boey, G. P. Maniam, S. A. Hamid, "Performance of calcium oxide as a heterogeneous catalyst in biodiesel production: A review", 
Chem. Eng. J. Bioch. Eng, vol. 168, pp. 15-22, 2011.

[19] G. E. Box, J. S. Hunter, W. G. Hunter, Estadistica para investigadores, diseño, innovación y descubrimiento, 2 ed. España: Reverté, 2008.

[20] E. I. Hervcot, E. E. Utgés, A. R. Farias, J. M. Delgado, M. R. Alonso, S. S. Rodríguez, R. Zambón, "Incertidumbre de medición en determinaciones químicas", En Jornadas IRAM, XXV, Trabajo TC-12, Disponible en: http://www.uniram.com.ar/jornadas/XXV/TC12.pdf.

[21] R. D. Chirico, M. Frenkel, V. V. Diky, K. N. Marsh, R. C. Wilhoit, "Thermomlsan XMLBased Approach for Storage and Exchange of
Experimental and Critically Evaluated Thermophysical and Thermochemical Property Data. 2. Uncertainties", J. Chem. Eng., vol. 48, pp. 1344-1359, 2003.

[22] T. F. Dossin, M. F. Reyniers, R. J. Berger, G. B. Marin, "Simulation of heterogeneously MgOcatalyzed transesterification for fine-chemical and biodiesel industrial production", Appl. $\mathrm{Ca}$ tal. B. Environ, vol. 67, pp. 136-148, 2006.

[23] B. J. Bravo, A. A. Botero, A. M. Botero, "El método de Newton-Rhapson - la alternativa del ingeniero para resolver sistemas de ecuaciones no lineales", Sci. Tech, vol. 27, pp. 221-224, 2005 . 